MATEC Web of Conferences 1, 01003 (2012)

DOI: $10.1051 /$ matecconf $/ 20120101003$

(C) Owned by the authors, published by EDP Sciences, 2012

\title{
On assessing the robustness of an input signal optimization algorithm for damage detection: the Info-Gap Decision Theory approach
}

\author{
M. Pasquali ${ }^{1, a}$, W. Lacarbonara ${ }^{1}$, C.J. Stull ${ }^{2}$, and C.R. Farrar ${ }^{2}$ \\ 1 Sapienza University of Rome, Via Eudossiana 18, 00184 Rome, Italy. \\ 2 Los Alamos National Laboratory, P.O. Box 1663, 87545 Los Alamos, NM, USA.
}

\begin{abstract}
The Info-Gap Decision Theory (IGDT) is here adopted to assess the robustness of a technique aimed at identifying the optimal excitation signal within a structural health monitoring (SHM) procedure. Given limited system response measurements and ever-present physical limits on the level of excitation, the ultimate goal of the mentioned technique is to improve the detectability of the damage increasing the difference between measurable outputs of the undamaged and damaged system. In particular, a 2 DOF massspring-damper system characterized by the presence of a nonlinear stiffness is considered. Uncertainty is introduced within the system under the form of deviations of its parameters (mass, stiffness, damping ratio...) from their nominal values. Variations in the performance of the mentioned technique are then evaluated both in terms of changes in the estimated difference between the responses of the damaged and undamaged system and in terms of deviations of the identified optimal input signal from its nominal estimation. Finally, plots of the performances of the analyzed algorithm for different levels of uncertainty are obtained, showing which parameters are more sensitive to the presence of uncertainty and thus enabling a clear evaluation of its robustness.
\end{abstract}

\section{Introduction}

Many developments have been carried out in the SHM field by the civil, aerospace and mechanical engineering communities, giving rise to new technologies which have improved upon and extended the application of these research fields [1]. In this sense, the engineer tasked with developing a SHM system has to address the difficulty of choosing from among these numerous technologies, doing it in a defensible manner. However, objective reasoning is not what usually leads the choice of one classifier over another, as the "tried-and-true" strategy is often the most addressed one. It can surely be inferred that relying on experienced solutions is often a robust decision, as the engineer does not expect to be surprised by the results associated with such a decision. The real drawback of such an approach is that it leaves little room for untried methodologies, which can potentially guarantee better performances. Information-Gap Decision Theory (IGDT) [2] goes beyond this subjective approach and offers a principled and hence, defensible manner of assessing the robustness of decisions to uncertainties affecting those decisions, in particular when there is little information available about these uncertainties a priori, rendering a probabilistic definition difficult to employ.

In this work, the robustness of a method aimed at designing excitation signals for the purpose of damage detection [3] is assessed via an IGDT-based approach. The proposed method is applied on a 2 DOF mass-spring-damper system characterized by the presence of a nonlinear stiffness. Uncertainty

a e-mail: michele.pasquali@uniroma1.it

This is an Open Access article distributed under the terms of the Creative Commons Attribution License 2.0, which permits unrestricted use, distribution, and reproduction in any medium, provided the original work is properly cited. 

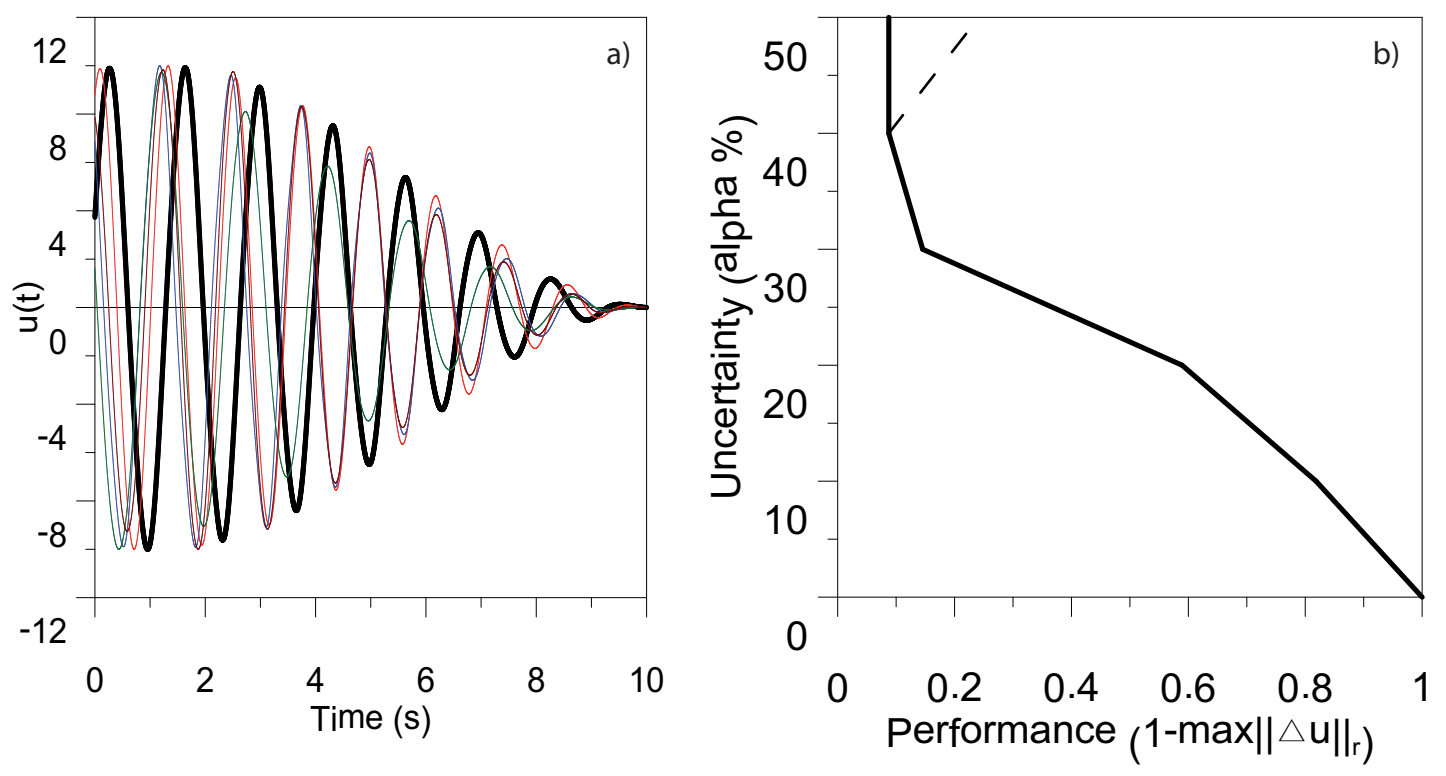

Fig. 1. Effects of uncertainty on the detected optimal excitation signal (a) and an uncertainty vs. performance curve (b)

is introduced within the system under the form of deviations of its parameters (mass, stiffness, damping ratio...) from their nominal values. Variations in the performance of the mentioned technique are then evaluated both in terms of changes in the estimated difference between the responses of the damaged and undamaged system and in terms of deviations of the identified optimal input signal from its nominal estimation. Finally, plots of the performances of the analyzed algorithm for different levels of uncertainty are obtained, showing which parameters are more sensitive to the presence of uncertainty and thus enabling a clear evaluation of its robustness.

\section{The Info-Gap Decision Theory approach}

Classically, uncertainty is represented by probability theory [4], which quantifies lack of information either in terms of the frequency of the recurrence of events, or in terms of an observer's subjective degrees of belief [5]. However, when the information is really scarce or, even if plentiful, the system is prone to fundamental change so that the past is a weak indication of the future, the need to classify uncertainty without using probability distribution functions arises. In these cases, in fact, in lieu of extensive resources of money and time needed to develop a well-grounded realistic model, and in light of unpredictable future non expected events, an approximated model is adopted, with the realization that it deviates by an unknown but possibly substantial margin from the actual behavior. In so doing, a large information gap between what is known and what needs to be known is acknowledged. This info-gap is a severe form of uncertainty and can be handled with an approach based on Info-Gap Decision Theory. The basic decision functions in Info-Gap Decision Theory are constituted by the so called immunity functions, i.e., the robustness and the opportuneness functions. The first expresses the greatest level of uncertainty at which failure cannot occur; the latter represents the least level of uncertainty which entails the possibility of sweeping success. In this sense, the immunity functions address, respectively, the pernicious and propitious facets of uncertainty: once the required performances of the system are defined, the info-gap-based approach provides the decision maker with an effective mean to evaluate the robustness and the opportuneness related to a particular decision, allowing him to assess whether that decision has to be taken or not. 


\section{Robustness and Uncertainty}

Uncertainty is introduced within the analyzed 2 DOF system under the form of a lack of knowledge involving the parameters which define the system itself, that is, a percent variation of the coefficients that appear in the governing equations of the problem with respect to their nominal values. The algorithm presented in [3] is used to identify the optimal excitation signals related to each value of uncertainty considered within the system. A performance index is then defined (a quantity representative of the point-wise convergence of the identified optimal excitation to the nominal optimal excitation signal), and uncertainty vs. performance curves are created, allowing the decision maker to asses the robustness of the analyzed algorithm to the presence of uncertainty.

\section{References}

1. H. Sohn, C.R. Farrar, et al., "A review of structural health monitoring literature from 1996 to 2001 ", Technical Report LA-13976-MS, Los Alamos National Laboratory (2004).

2. Y. Ben-Haim, "Info-Gap Decision Theory: Decisions Under Severe Uncertainty ", 2nd ed. Oxford: Academic Press, Oxford (1980).

3. M.T. Bement, T.R. Bewley, "Excitation design for damage detection using iterative adjoint-based optimization - Part1: Method development ", Mechanical Systems and Signal Processing, 23 (2009), pp.783-793.

4. A.N. Kolmogorov, "Fundations of the theory of probability", Chelsea Publishing, Oxford (1950).

5. R. von Mises, "Probability, Statistics and Truth ", Dover Pubblications, London (1981).

6. Y. Ben-Haim, "Robust Reliability in the Mechanical Sciences ”, pringer-Verlag, Berlin (1996). 